\title{
Variable Gravity Field and Throughflow Effects on Penetrative CONVECTION IN A POROUS LAYER
}

\author{
Gangadharaiah, Y. H., Suma, S. P., Ananda, K. \\ Department of Mathematics, \\ New Horizon College of Engineering, \\ Bangalore-560 103, India \\ E-mail address: gangu.honnappa@gmail.com
}

\begin{abstract}
The effect of vertical throughflow and variable gravity field on the onset of penetrative convection simulated via internal heating in a porous medium is studied. Flow in the porous medium is governed by Forchheimer-extended Darcy equation. The boundaries are considered to be rigid, however permeable, and insulated to temperature perturbations. The eigen value problem is solved using a regular perturbation technique with wave number as a perturbation parameter. The variable gravity parameter, the direction of throughflow and the presence of volumetric internal heat source in a porous layer play a decisive role on the stability characteristics of the system. In addition, the influence of Prandtl number arising due to throughflow is also emphasized on the stability of the system. It is observed that both stabilizing and destabilizing factors can be enhanced due to the simultaneous presence of a volumetric source, gravity field and vertical throughflow so that a more precise control (suppress or augment) of thermal convective instability in a layer of porous medium is possible.
\end{abstract}

Key words: penetrative convection; gravity field; throughflow.

\section{Council for Innovative Research}

Peer Review Research Publishing System

Journal: INTERNATIONAL JOURNAL OF COMPUTERS \& TECHNOLOGY

Vol 5, No 3

editor@cirworld.com

www.cirworld.com, member.cirworld.com 


\section{Introduction}

The convective instability in a fluid-saturated porous medium due to a vertical throughflow is of relevance in the study of geothermal activities, underground transport of pollutants, gas reservoirs, groundwater pollution and thermal insulation, in the manufacture of composite materials used in aircraft structures and automobile industries, geophysical systems, crystal growth and in flow in biological materials. Copious literature available on this topic is well documented in the book by Nield and Bejan (2006). The in situ processing of energy resources such as coal, oil shale, or geothermal energy often involves the throughflow in the porous medium. The importance of buoyancy-driven instability in such systems may become significant when precise processing is required (Sutton 1970; Homsy and Sherwood 1976; Nield 1987; Khalili and Shivakumara (1998; 2003); Shivakumara and Nanjundappa 2006; Hill 2007; Hill et al. 2007; Barletta et al.2010; Nield and Kuznetsov 2011).

There are situations of great practical importance where the porous material offers its own source of heat. This gives a different way in which a convective flow can be set up through the local heat generation within the porous media. Such a situation can occur through radioactive decay or through, in the present perspective, a relatively weak exothermic reaction which can take place within the porous material. To be more specific, internal heat is the main source of energy for celestial bodies caused by nuclear fusion and decaying of radioactive materials, which keeps the celestial objects warm and active. It is due to the internal heating of the earth that there exists a thermal gradient between the interior and exterior of the earth's crust, saturated by multi components fluids, which helps convective flow, thereby transferring the thermal energy toward the surface of the earth. Therefore, the role of internal heat generation becomes very important in several applications that include geophysics, reactor safety analyses, metal waste form development for spent nuclear fuel, fire and combustion studies, and storage of radioactive materials. Penetrative convection in porous media has received great attention during the past few decades because of the importance of this process which occurs in many engineering and natural systems of practical interest such as geothermal energy utilization, thermal energy storage and recovery systems, petroleum reservoirs, industrial and agricultural water distribution, to name just a few applications. Very recent reviews by Nield and Bejan 2006; Carr 2004; Capone, Carr and Putter 2003; Hill 2004; Straughan 2008; Tse and Chasnov 1998; Straughan and Walker 1976; and Zhang and Schubert 2002.

The assumption of constant gravity in the problems of astrophysics and geophysics are usually far removed from the reality and our endeavour is to look into the simple extension of the plane layer problem to include variable gravitational field. Although the investigation of thermal instability due to a time dependent gravity field has attracted the attention of several researchers (Saunders et al. 1992; Clever et al.1993; Rees and Pop. 2000)convection due to a gravity field varying with distance, has received little attention. However, in studying large scale flows in the Earth's crust it is imperative to consider the gravitational field of the earth as a variable quantity, varying with distance from the centre, as pointed out by Pradhan and Samal (1987). The existence of elevated gravity levels varying with distance, during the formation of crystals grown from the molten phase in a furnace placed at the arm extremity of a centrifuge, have been reported by Rodot et al. (1990). Chen and Chen (1992) have investigated the effect of such a gravity gradient on the onset of salt finger convection in a pure fluid. The thermal stability of a non-viscous fluid layer and that of a viscous fluid layer, subject to a gravitational field varying with distance in the layer, have been analysed by Pradhan and Samal (1987) and Straughan(1989) respectively. The linear and nonlinear stability limits for convection in an isotropic porous medium with internal heat source under variable gravity fields have been obtained by Rionero and Straughan (1990) and Kaloni and Qiao (2001). Prakash and Raj (1996) have studied the Rayleigh-Taylor instability of partially ionized plasma in the presence of variable gravitational field in porous medium. Alex and Patil (1999) have investigated the convective instability of a horizontal fluid-saturated porous layer with inclined temperature gradient subject to a gravitational field varying linearly with distance in the layer. Alex and Patil (2002a) have investigated the effect of internal heat source and inclined temperature gradient on convection in an porous medium subjected to gravity field. Recently Govender (2005) has made stability analyses to investigate the effect of low amplitude gravity modulation on convection in a homogeneous porous layer heated from below.

The intent of the present study is to obtain the criterion for the onset of penetrative convection in a fluid-saturated porous medium in the presence of vertical throughflow and gravitational field via internal heating. The boundaries are considered to be insulated to temperature perturbations. A regular perturbation technique with wave number as $a$ perturbation parameter is used to solve the eigen value problem in a closed form. A wide-ranging parametric study is undertaken to explore their impact on the stability characteristics of the system.

\section{Mathematical Formulation}

The physical configuration consists of an infinite horizontal incompressible fluid-saturated porous layer of thickness $d$ with throughflow of vertical velocity $W_{0}$ and the $z$-axis pointing vertically upwards opposing the direction of gravity. The both upper and lower boundary is insulated to temperature 
perturbations. The temperatures of the lower and upper boundaries are taken to be uniform and equal to $T_{l}$ and $T_{u}$ respectively, with $T_{l}>T_{u}$. The density of the fluid is given by

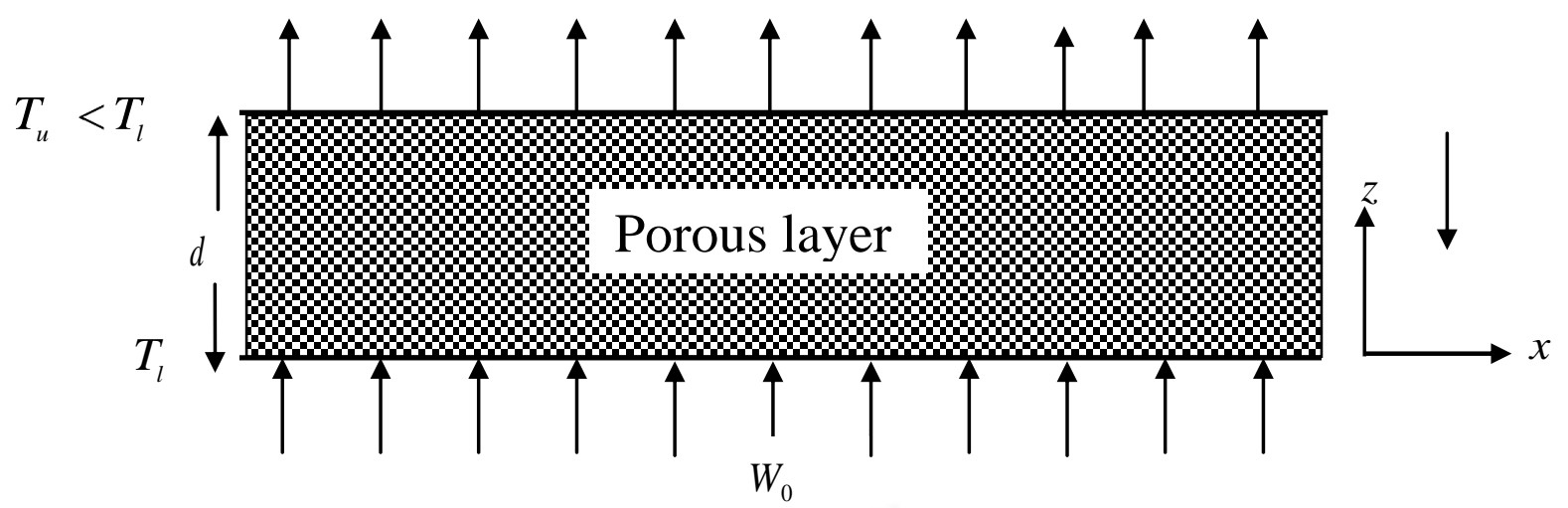

Fig.1 Physical configuration

$\rho=\rho_{0}\left[1-\alpha T-T_{0}\right]$

where $\alpha$ is the positive coefficient of the thermal liquid expansion and $\rho_{0}$ is the value at the reference temperature $T_{0}$

As pointed by Beck (1972), the inclusion of inertial effects in the porous medium by adding $\left(\vec{V}_{m} \cdot \nabla\right) \vec{V}_{m}$ is not correct for the reasons that this term (i) vanishes identically if the flow is unidirectional and hence cannot represent the inertial effect(increase in drag) in that case, and (ii) raises the order of the differential equation with respect to space derivatives when the base flow is not quiescent which leads to inconsistency with the velocity boundary conditions when the Darcy law is employed. For many naturally occurring porous media, Nield and Joseph (1985) have shown that $\left|\vec{V}_{m}\right| \vec{V}_{m}$ is the appropriate inertia term in the momentum equation, which implies that the effect of inertia is a drag term quadratic in the velocity $\vec{V}_{m}$. These aspects are discussed at length by Nield and Bejan (2006). Under the circumstances, the Darcy-Forchheimer equation is used to describe the flow in the porous medium to account for inertia effects arising due to vertical throughflow. Thus, the governing equations for the porous layer in the Boussinesq approximation are:

$$
\begin{aligned}
& \nabla \cdot \vec{V}=0 \\
& \frac{\rho_{0}}{\phi}\left(\frac{\partial \vec{V}}{\partial t}+\frac{C_{b}}{\sqrt{\kappa}} \vec{V}|\vec{V}|\right)=-\nabla p-\frac{\mu}{\kappa} \vec{V}+\rho \vec{g}
\end{aligned}
$$

$A \frac{\partial T}{\partial t}+\vec{V} \cdot \nabla T=k \nabla^{2} T+q$

where, following Chen and Chen (1992) it has been assumed that the gravity vector $\vec{g}$ varies linearly with distance. That is,

$\vec{g}=-g_{0} 1+\lambda z \hat{k}$

Where $\lambda$, the variable gravity coefficient is assumed to be a constant.

In the above equations, $\vec{V}=u, v, w$ is the velocity vector, $T$ is the temperature, $\mu$ is the dynamic viscosity, $p$ is the pressure, $q$ is the heat source in the porous layer, $C_{b}$ is the form drag constant, $A$ is the 
ratio of heat capacities, $\rho_{0}$ is the reference fluid density, $\kappa$ and $\phi$ permeability and porosity of the porous medium.

The basic steady state solution is of the form

$$
u, v, w, p, T=0,0, w_{0}, p_{b}(z), T_{b}(z)
$$

where the subscript $b$ denotes the basic state and the temperature distributions in the basic state is given by

$$
T_{b} \quad z=T_{l}+\frac{q d}{W_{0}}\left[\left(\frac{z}{d}+\frac{1-e^{W_{0} z / \kappa}}{e^{W_{0} d / \kappa}-1}\right)\right]-T_{l}-T_{u}\left(\frac{1-e^{W_{0} z / \kappa}}{e^{W_{0} d / \kappa}-1}\right)
$$

In order to investigate the stability of the basic solution, infinitesimal disturbances are introduced in the form

$$
u, v, w, p, T=0,0, w_{0}, p_{b}(z), T_{b}(z)+u^{\prime}, v^{\prime}, w^{\prime}, p^{\prime}, T^{\prime}
$$

where the primed quantities are the perturbation and assumed to be small. Now Eq.(3.8)is substituted in Eqs.(2) - (4) and linearized in the usual manner. The pressure term is eliminated from Eq. (3) by taking curl twice on this equation and only the vertical component is retained. The variables are then nondimensionalized using $d, d^{2} / \kappa, \kappa / d$ and $T_{l}-T_{u}$ units of length, time, velocity, and temperature. The non-dimensional disturbance equations are now given by

$$
\begin{aligned}
& {\left[\frac{D a}{\phi \operatorname{Pr}}\left(\frac{\partial}{\partial t}+C_{b m}|P e|\right)+1\right] \nabla^{2} w=R 1+\lambda z \nabla^{2} w} \\
& {\left[\frac{\partial}{\partial t}+P e \frac{\partial}{\partial z}-\nabla^{2}\right] T=\left[\frac{2 N s}{P e}+(P e+2 N s) \frac{e^{P e z}}{1-e^{P e}}\right] w}
\end{aligned}
$$

where $R=\alpha g_{0} \quad T_{l}-T_{u} d^{3} / v \kappa$ is the Rayleigh number, $N s=q d^{2} / 2 \kappa T_{l}-T_{u}$ is the dimensionless heat source strength, $D a=K / d^{2}$ is the Darcy number and $C_{b m}=C_{b} d / \sqrt{K}$ is the nondimensional group.

The principle of exchange instabilities holds good even for the present configuration as well, hence the time derivatives will be dropped conveniently from Eqs. (9) and (10). Then performing a normal mode expansion and seek solutions for the dependent variables as

$$
w, T=\left[\begin{array}{llll}
W & z & \Theta & \Theta
\end{array}\right] \exp [i l x+m y]
$$

and substituting them in Eqs. (9)and(10) (with $\partial / \partial t=0$ ), we obtain the following ordinary differential equations

$$
\begin{aligned}
& 1+G \quad D^{2}-a^{2} \quad W=-R a^{2} 1+\lambda z \Theta \\
& D^{2}-P e D-a^{2} \Theta=f(z) W
\end{aligned}
$$

Where $W$ is the amplitude of perturbed vertical velocity and $\Theta$ is the amplitude of perturbed temperature. In the above equations, $D=d / d z, a=\sqrt{l^{2}+m^{2}}$ is the overall horizontal wave numbers, $G=C_{b m} D a|P e| / \phi P r_{m}$ is the nondimensional groups and

$f(z)=\frac{2 N s}{P e}+\frac{P e+2 N s}{1-e^{P e}} e^{P e z}$.

The boundary conditions take the form

$$
W=0 \text { or } \quad D W=0 \quad \text { at } \quad z=0,1
$$


$D \Theta=0 \quad$ at $\quad z=0,1$.

\section{Method of Solution}

When the boundaries are insulated to temperature perturbations, the critical wave number is vanishingly small. Hence, the solution of eigen value problem may be obtained in a closed form using regular perturbation technique with wave number $a$ as the perturbation parameter. The variables are expressed in a series solution of the form

$$
W, \Theta=W_{0}, \Theta_{0}+a^{2} W_{1}, \Theta_{1}+--------
$$

Substituting Eq. (17) in to Eqs. (12)-(13) and collecting terms of zeroth -order we get

$$
\begin{aligned}
& 1+G \quad D^{2} W_{0}=0 \\
& D^{2} \Theta_{0}-P e D \Theta_{0}=f(z) W_{0} .
\end{aligned}
$$

Then solutions to the above equations are $W_{0}=0$ and $\Theta_{0}=1$

The first-order equations are

$$
\begin{gathered}
1+G \quad D^{2} W_{1}=-R(1+\lambda z) \\
D^{2} \Theta_{1}-P e D \Theta_{1}=1+f(z) W_{1} .
\end{gathered}
$$

The general solution of Eq. (21) is

$W_{1}=\frac{-R}{(1+G)}\left(\frac{z^{2}}{2}+\lambda \frac{z^{3}}{6}\right)+c_{1} z+c_{2}$

From solvability condition, we have

$\left\langle 1+f(z) W_{1}\right\rangle=0$

The symbol $(\ldots)$ denotes integration from 0 to 1 .

The expressions for $W_{1}$ is substituted into Eq. (24) and integrated to yield an expression for the critical Rayleigh number $R_{c}$ for different types of boundaries.

\subsection{Both Boundaries are Impermeable and Insulating}

The boundary conditions are;

$$
W_{1}=0, D \Theta=0 \quad \text { at } \quad z=0,1 \text {. }
$$

Hence the values of $c_{1}$ and $\mathrm{c}_{2}$ are

$$
c_{1}=\frac{R}{1+G}\left(\frac{1}{2}+\frac{\lambda}{6}\right), c_{2}=0 .
$$

Then expression for $R_{c}$ is given by 


$$
R_{c}=\frac{121+G P e^{4} e^{P e}-1}{\left[\begin{array}{lll}
-e^{P e}-1 & 3 N s+2 \lambda P e^{3}+2 e^{P e}(2 N s+P e)-6 \lambda(P e-\lambda)-\lambda P e & 6 e^{P e}+(\lambda+3) P e
\end{array}\right]} .
$$

In the absence of internal heating i.e $N s \rightarrow 0$, the above expression reduces to

$$
R_{c}=\frac{61+G P e^{3}}{\left[3+2 \lambda P e^{2} \operatorname{coth} P e / 2-6(P e-\lambda)-\lambda P e \frac{6 e^{P e}+P e}{e^{P e}-1}\right]} .
$$

In the absence of gravitational field i.e $\lambda \rightarrow 0$, the above expression reduces to

$$
R_{c}=\frac{21+G P e^{2}}{[P e \operatorname{coth} P e / 2-2]}
$$

and when Darcy equation is used to describe the porous medium i.e $G \rightarrow 0$, the above expression reduces to that of Nield(1987).

$$
R_{c}=\frac{2 P e^{2}}{[P e \operatorname{coth} P e / 2-2]} \text {. }
$$

In the absence of throughflow i.e $P e \rightarrow 0$, Eq. (29) reduces to $R_{c}=12$, which is known exact value.

\section{2. Lower Boundary Impermeable and Insulating, Upper Boundary Porous and Insulating}

The boundary conditions are;

$$
\begin{aligned}
& W_{1}=0, D \Theta=0 \quad \text { at } \quad z=0 \\
& D W_{1}=0, D \Theta=0 \quad \text { at } \quad z=1 .
\end{aligned}
$$

Hence the values of $c_{1}$ and $\mathrm{c}_{2}$ are

$$
c_{1}=\frac{R}{1+G}\left(1+\frac{\lambda}{2}\right), c_{2}=0
$$

Then expression for $R_{c}$ is given by

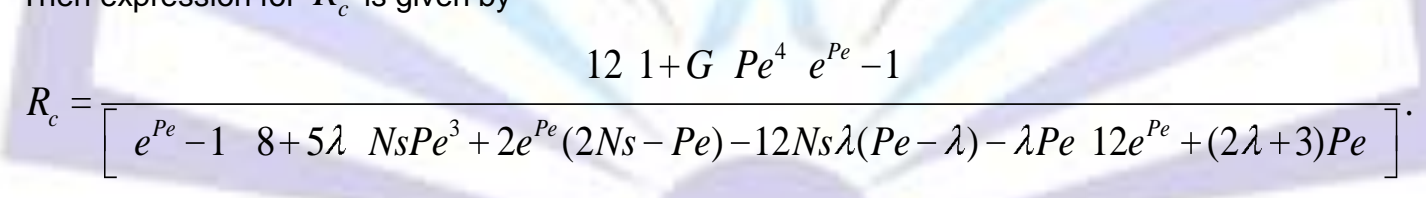

In the absence of internal heating i.e $N s \rightarrow 0$, the above expression reduces to

$$
R_{c}=\frac{61+G P e^{3} e^{P e}-1}{\left[3+2 \lambda P e^{3} e^{P e}-6(1+\lambda) P e e^{P e}+6 \lambda e^{P e}+32+\lambda P e^{2}+6 P e-\lambda\right]} .
$$

In the absence of heat source i.e $\lambda \rightarrow 0$, the above expression reduces to

$$
R_{c}=\frac{21+G P e^{2} e^{P e}-1}{\left[e^{P e}\left(P e^{2}-2\right)+2 P e+2\right]}
$$

and when Darcy equation is used to describe the porous medium i.e $G \rightarrow 0$, the above expression reduces to that of Nield (1987) 


$$
R_{c}=\frac{2 P e^{2} e^{P e}-1}{\left[e^{P e}\left(P e^{2}-2\right)+2 P e+2\right]}
$$

In the absence of throughflow i.e $P e \rightarrow 0$, Eq. (35) reduces to $R_{c}=3$, which is known exact value.

\subsection{Lower Boundary Porous and Insulating, Upper Boundary Impermeable and Insulating}

The boundary conditions are;

$$
\begin{aligned}
& D W_{1}=0, D \Theta=0 \quad \text { at } z=0 \\
& W_{1}=0, D \Theta=0 \quad \text { at } \quad z=1 .
\end{aligned}
$$

Hence the values of $c_{1}$ and $c_{2}$ are

$$
c_{1}=\frac{R}{1+G}\left(1+\frac{\lambda}{2}\right), c_{2}=0 .
$$

Then expression for $R_{c}$ is given by

$$
R_{c}=\frac{121+G P e^{4} e^{P e}-1}{\left[\begin{array}{ll}
e^{P e}-1 & 8+3 \lambda N s P e^{3}+2 e^{P e}(4 N s+2 P e)+12 N s \lambda\left(3+P e^{2}+6 \lambda\right)+12 e^{P e}+(2 \lambda+3) P e
\end{array}\right]} .
$$

In the absence of internal heating i.e $N s \rightarrow 0$, the above expression reduces to

$$
R_{c}=\frac{61+G P e^{3} e^{P e}-1}{\left[32+\lambda P e^{2} e^{P e}-6(1+\lambda) P e e^{P e}+6 \lambda e^{P e}-3+\lambda P e^{3}+6 P e-\lambda\right]} .
$$

In the absence of gravitational field i.e $\lambda \rightarrow 0$, the above expression reduces to

$$
R_{c}=\frac{21+G P e^{2} e^{P e}-1}{\left[2 e^{P e}(P e-1)-P e^{2}+2\right]} \text {. }
$$

In the absence of throughflow i.e $P e \rightarrow 0$, Eq. (40) reduces to $R_{c}=3$, which is known exact value.

The expression for $R_{c}$ is evaluated for different values of various physical parameters and the results are discussed in detail in the next section.

\section{Results and Discussion}

The onset of penetrative convection via internal heating in the presence of a vertical throughflow and variable gravity field is considered in a system consisting of a porous layer. In the calculation, we have chosen the value of $\phi=0.389, C_{b}=209.25$ and $\sqrt{D a}=3.04 \times 10^{-3}$ which correspond to $3 \mathrm{~cm}$ deep porous layer consisting of $3 \mathrm{~mm}$ diameter glass beads.

\section{Case (1) Both Boundaries are Impermeable and Insulating}

(a)Absence of Variable Gravity $(\lambda=0)$

The critical Rayleigh number computed from Eqs. (26) and (33) in the absence of variable gravity field (i.e., $\lambda=0$ ) and internal heating(i.e., $N s=0$ ) are compared with those obtained from Nield (1987) in Table. 1 for two types of boundary conditions and the results are found to be in good agreement, when Darcy equation is used in the porous layer. When $N s=0, \lambda=0$ and $P e=0$, the known exact value 
$R_{c}=12$ (Nield 1987) is retrieved. The direction of throughflow has no influence on the stability of the system in the absence of internal heating and throughflow is to delay the onset of convection. This may be attributed to the fact that the primary effect of throughflow is to confine significant thermal gradients to a thermal boundary layer at the boundary toward which the throughflow is directed. The effective length scale is thus smaller than the layer thickness $d$ and hence the effective Rayleigh number which will be depending on $d^{3}$ will be much less than the actual Rayleigh number. A larger value of Rayleigh number is thus necessary to initiate the convection. Nonetheless, the simultaneous presence of throughflow and internal heating alters the basic temperature distribution so that the direction of throughflow also affects the stability of the system. As a result of this some unusual behaviours are observed namely, (i) downward throughflow initially shows some destabilizing effect (Fig. 2) and (ii) increasing internal heat source strength causes stabilizing effect for upward throughflow initially (Fig.3).

\section{(b)Presence of Variable Gravity $(\lambda \neq 0)$}

Figures 4 and 5 exhibit plots of $R_{c}$ as a function of $P e$ for two values of variable gravity parameter $\lambda=-1,1$ respectively with three values of Prandtl number $\operatorname{Pr}=0.1,1$ and 100 . From Figs. 4 and 5 it is observed that for all values of $\mathrm{Pr}$, both upward and downward throughflow stabilizes the system in the absence of internal heating $(N s=0)$. In the presence of internal heating $(N s=5)$ upward throughflow stabilizes the system for all values of $\mathrm{Pr}$ while downward throughflow destabilizes the system when $-4.6 \leq P e \leq 0,-5.2 \leq P e \leq 0,-5.4 \leq P e \leq 0-2.4 \leq P e \leq 0,-3.6 \leq P e \leq 0,-3.7 \leq P e \leq 0 f$ -or $\operatorname{Pr}=0.1,1$ and 100 , when $\lambda=-1,1$ respectively, and for higher values of $P e$ the system is stabilizing. And also from Figs 4 and 5 ,it can be observed that, $R_{c}$ is maximum when the variable gravity parameter $\lambda$ takes the value -1 . This is because, as noted by Chen and Chen (1992) when $\lambda<0$, the buoyancy effects are maximum at the lower wall and decrease with height. Further, the critical Rayleigh number is based on the gravity level at the lower wall. Thus when $\lambda<0$, the gravity level in the layer is decreased, which thereby causes an increase in the critical Rayleigh number. When $\lambda$ is positive the opposite effect is seen. That is, the critical Rayleigh number is less than that for the constant gravity case $\lambda=0$.

The effect of the variable gravity parameter $\lambda$ and the dimensionless heat source $N s$, on the critical Rayleigh number $R_{c}$ is illustrated in Fig.6 for $\operatorname{Pr}=10$, and $P e=3,-3$. From Fig. 6 it is observed that increasing internal heat source strength causes stabilizing effect for upward throughflow initially for all three values of gravity parameter $\lambda$ and downward throughflow destabilizes the system for all three values of gravity parameter $\lambda$. 


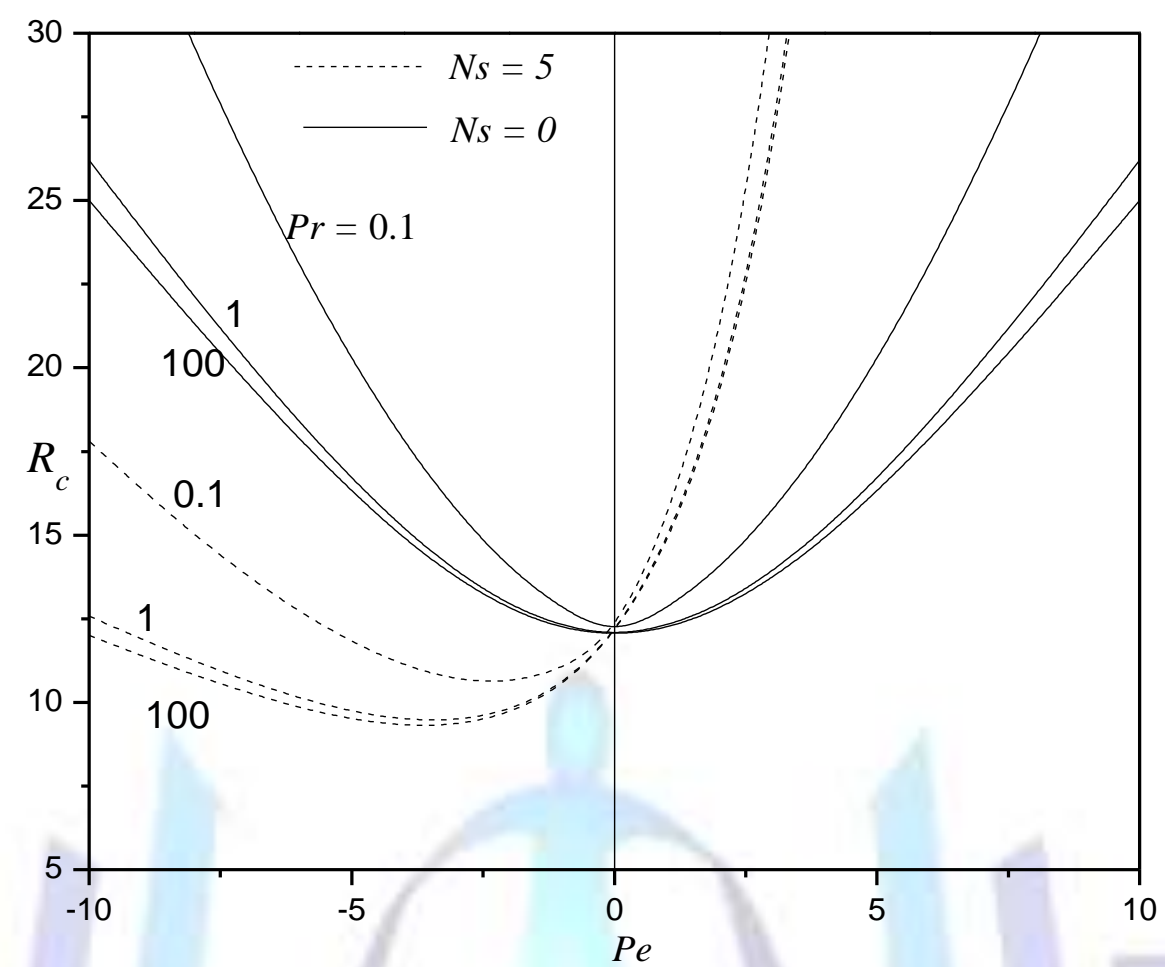

Fig.2 Critical Rayleigh number versus $P e$ for different values of $\operatorname{Pr}$ and $N s$ with $\lambda=0$ for both boundaries impermeable and insulating.

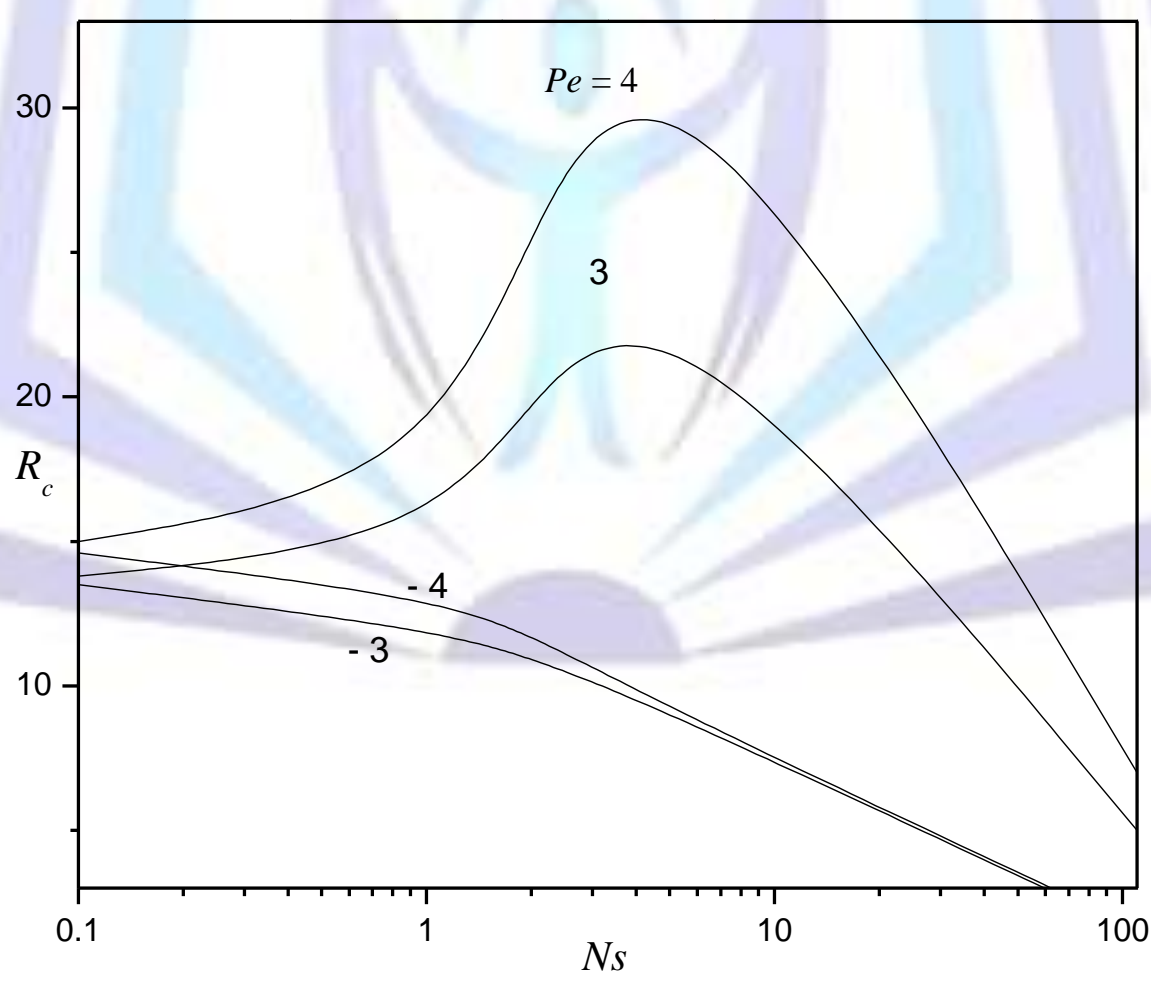

Fig. 3 Critical Rayleigh number versus $N s$ for different values of $P e$ with $\operatorname{Pr}=10, \lambda=0$ for both are boundaries impermeable and insulating. 


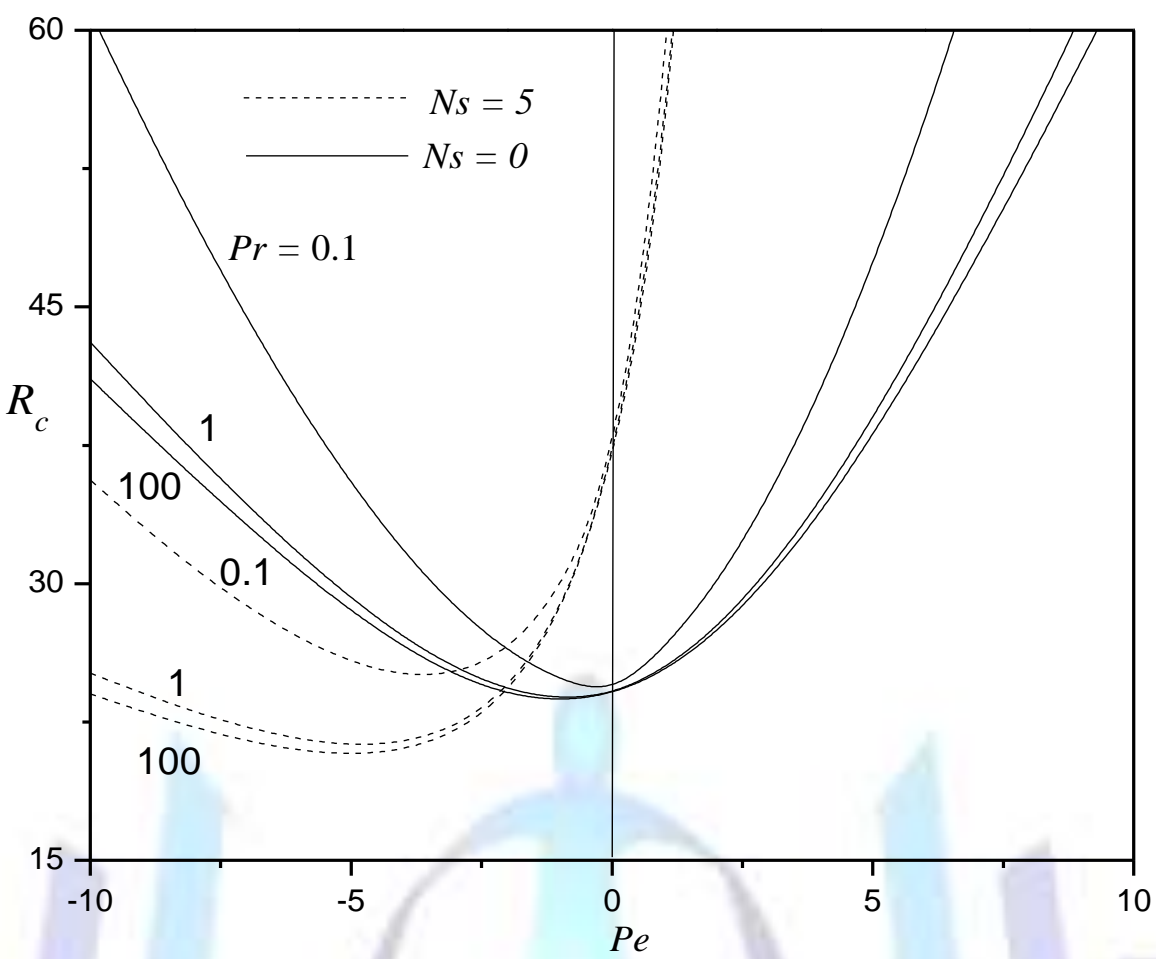

Fig. 4 Critical Rayleigh number versus $P e$ for different values of $\operatorname{Pr}$ and $N s$ with $\lambda=-1$ for both boundaries impermeable and insulating.

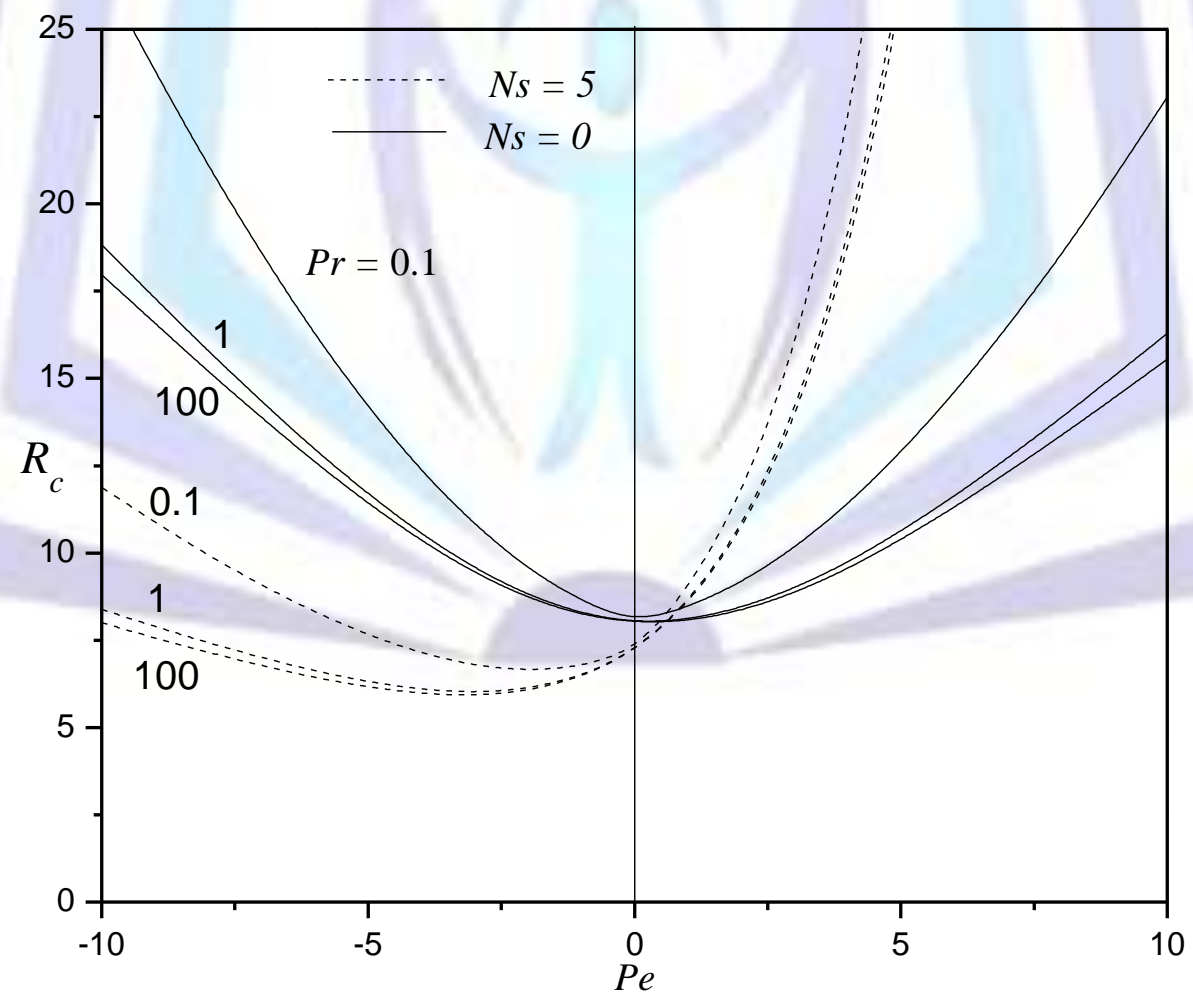

Fig.5 Critical Rayleigh number versus $P e$ for different values of $\operatorname{Pr}$ and $N s$ with $\lambda=1$ for both boundaries impermeable and insulating. 


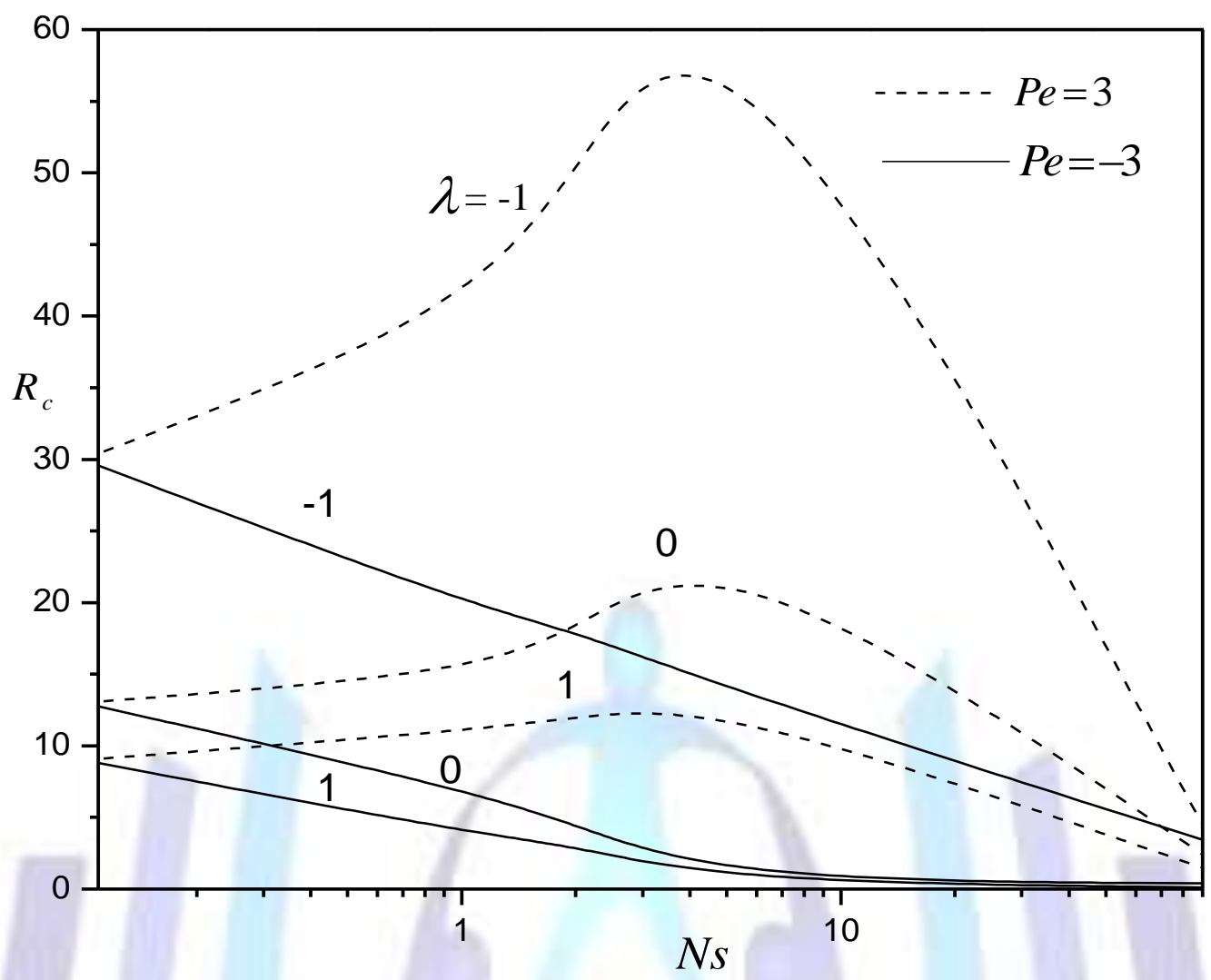

Fig. 6 Critical Rayleigh number versus $N s$ for different values of $\lambda$ and $P e$ with $P r=10$ for both are boundaries impermeable and insulating.

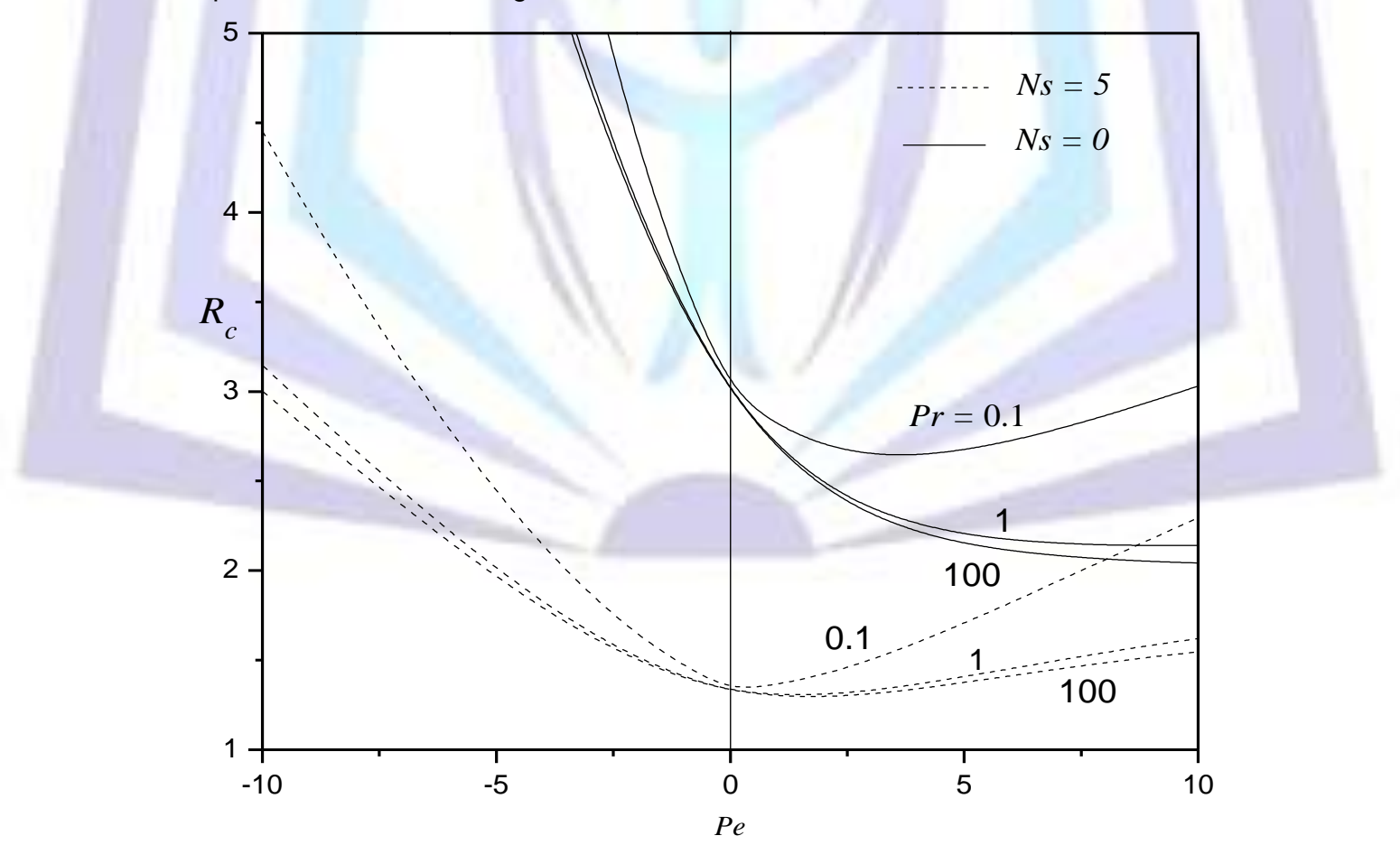

Fig.7 Critical Rayleigh number versus $P e$ for Different values of $\operatorname{Pr}$ and $N s$ with $\quad \lambda=0$ for lower boundary impermeable and insulating, upper boundary porous and insulating. 


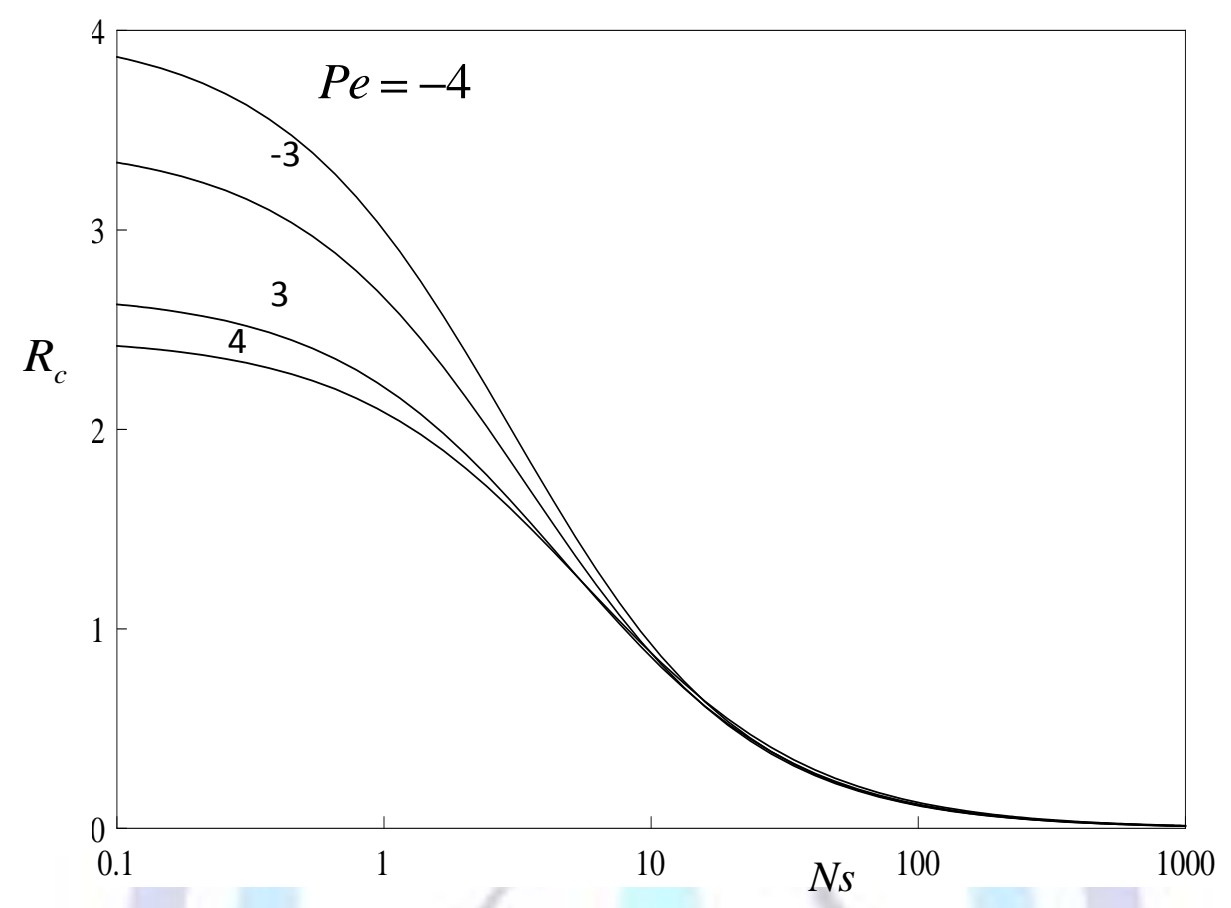

Fig. 8 Critical Rayleigh number versus $N s$ for different values of $P e$ with $\operatorname{Pr}=10, \lambda=0$ for lower boundary impermeable and insulating, upper boundary porous and insulating.

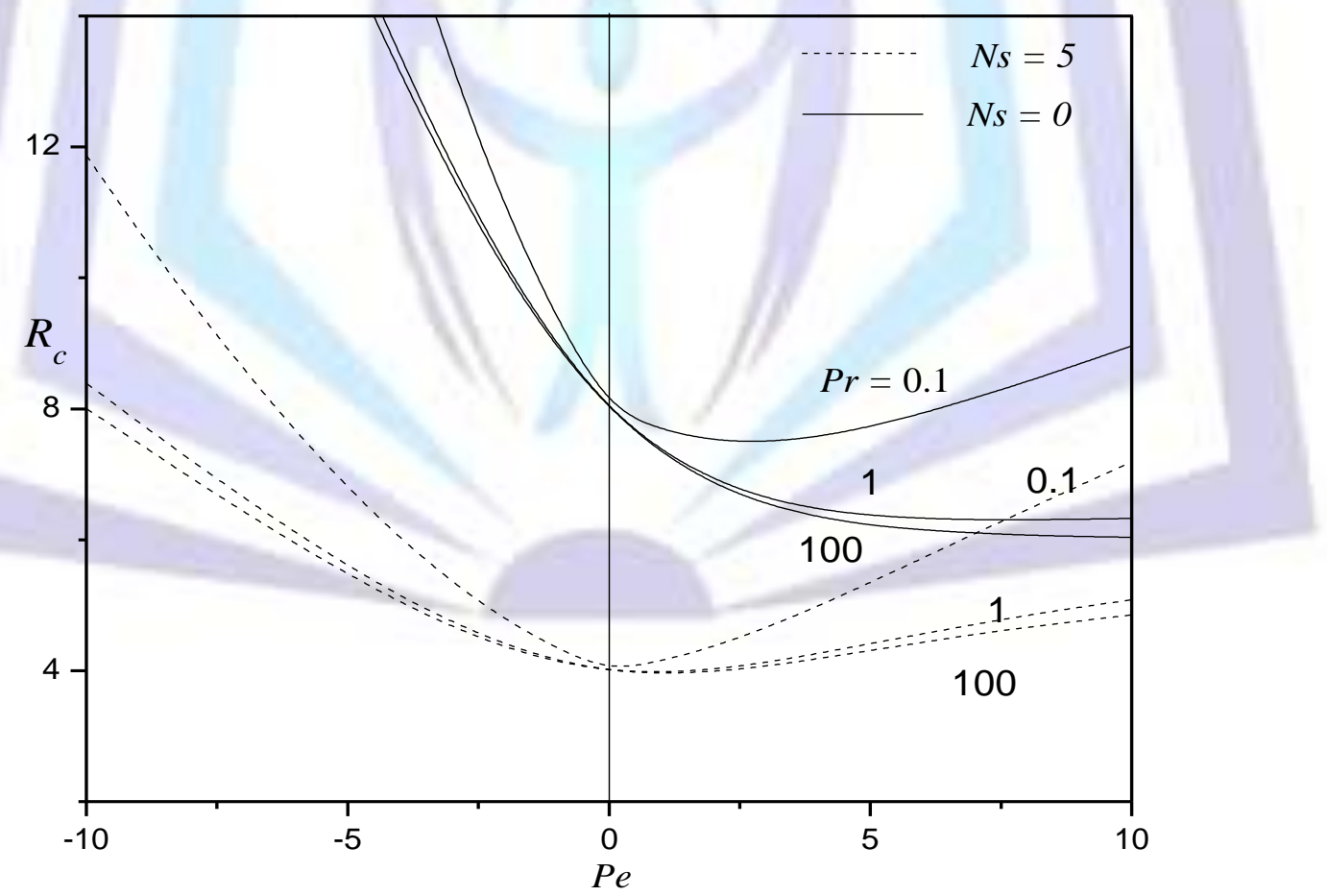

Fig.9 Critical Rayleigh number versus $P e$ for different values of $\operatorname{Pr}$ and $N s$ with $\lambda=-1$ for lower boundary impermeable and insulating, upper boundary porous and insulating. 


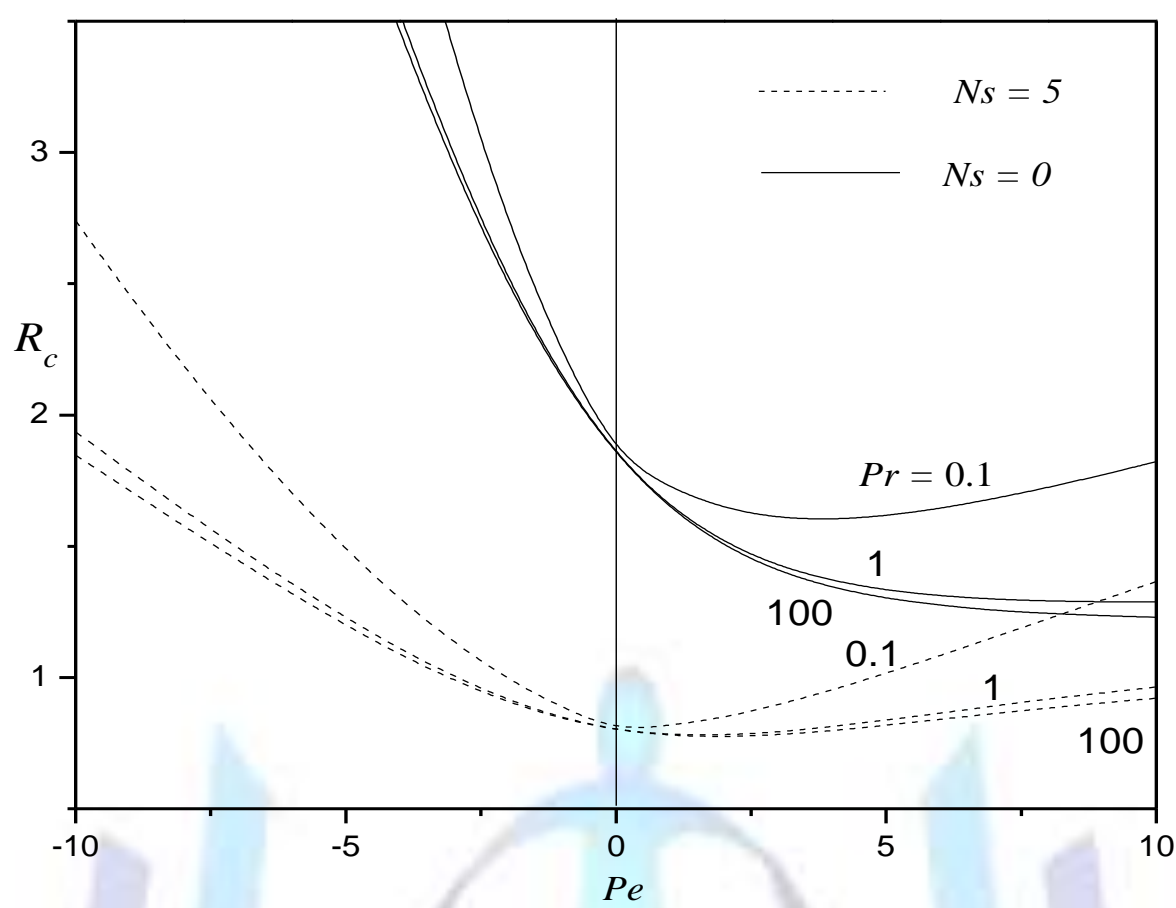

Fig.10 Critical Rayleigh number versus $P e$ for different values of $\operatorname{Pr}$ and $N s$ with $\lambda=1$ for lower boundary impermeable and insulating, upper boundary porous and insulating.



Fig. 11 Critical Rayleigh number versus $N s$ for different values of $\lambda$ and $P e$ with $P r=10$ for lower boundary impermeable and insulating, upper boundary porous and insulating. 


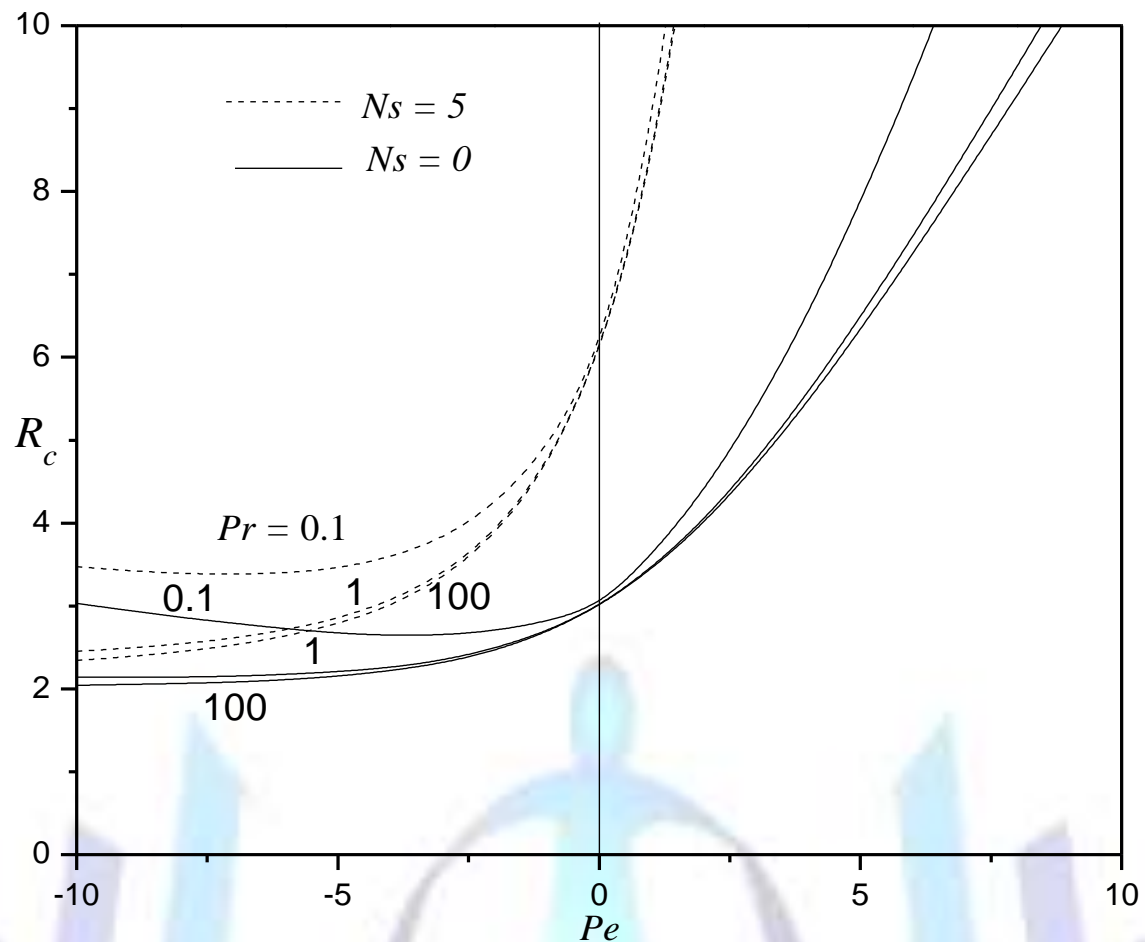

Fig.12 Critical Rayleigh number versus $P e$ for different values of $\operatorname{Pr}$ and $N s$ with $\lambda=0$ for lower boundary porous and insulating, upper boundary impermeable and insulating.

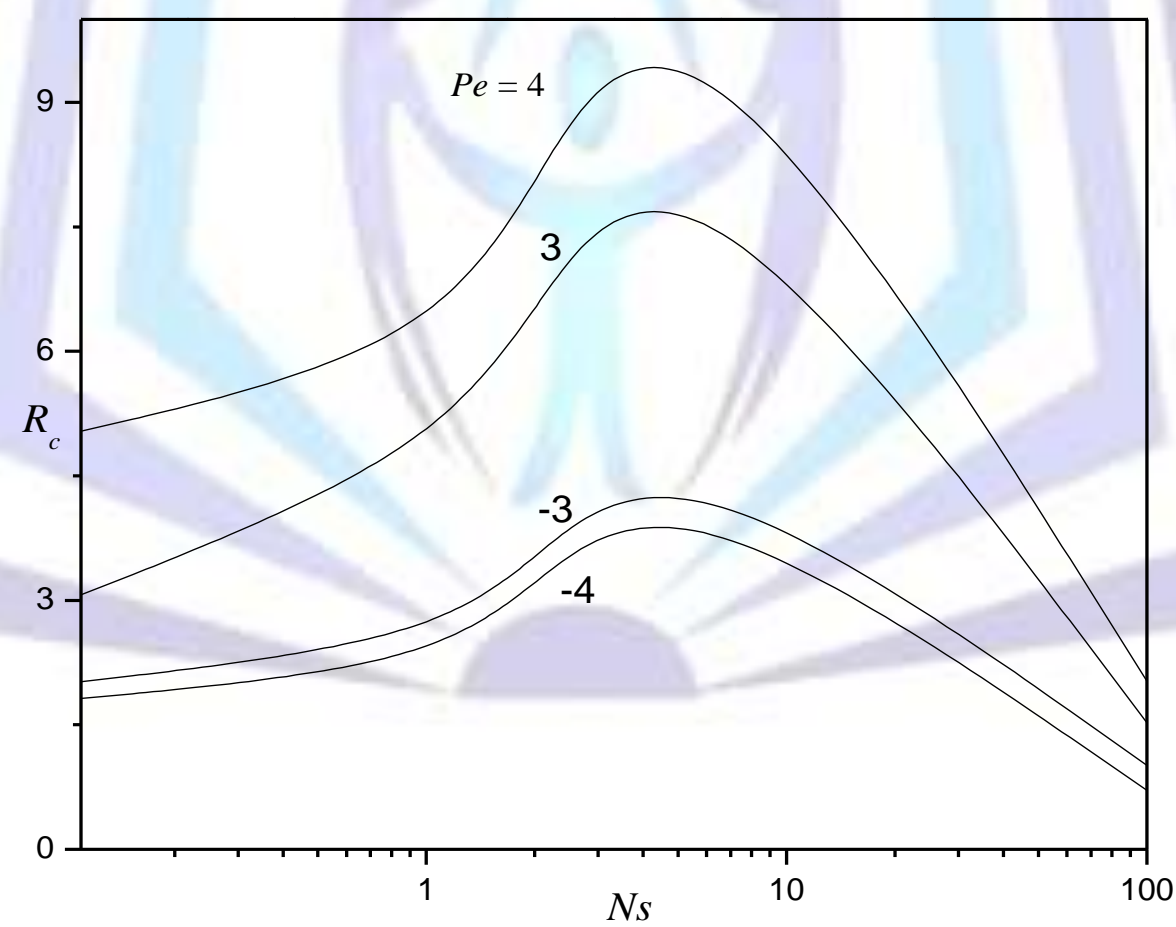

Fig. 13 Critical Rayleigh number versus $N s$ for different values of $P e$ with $P r=10, \lambda=0$ for lower boundary porous and insulating, upper boundary impermeable and insulating. 


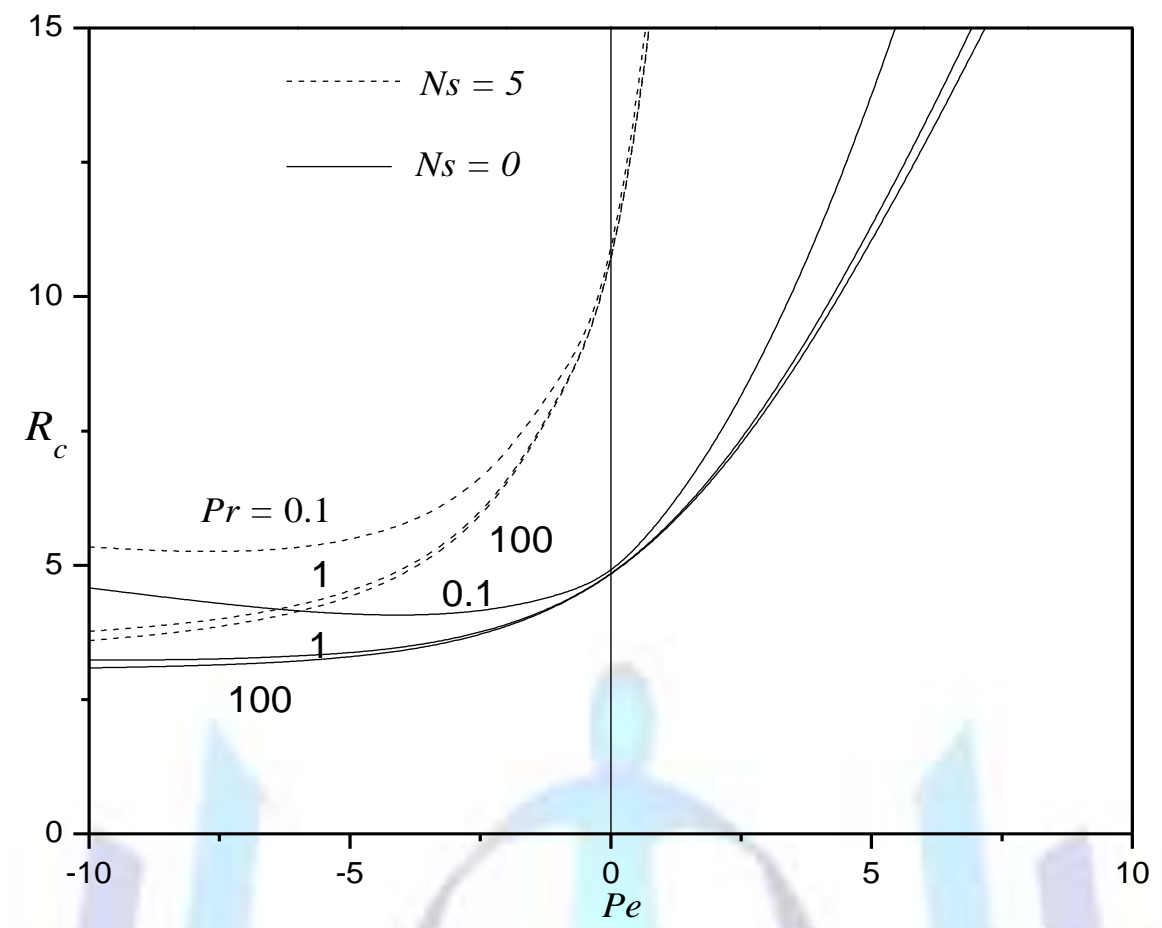

Fig.14 Critical Rayleigh number versus $P e$ for different values of $\operatorname{Pr}$ and $N s$ with $\lambda=-1$ for Lower boundary porous and insulating, upper boundary impermeable and insulating.

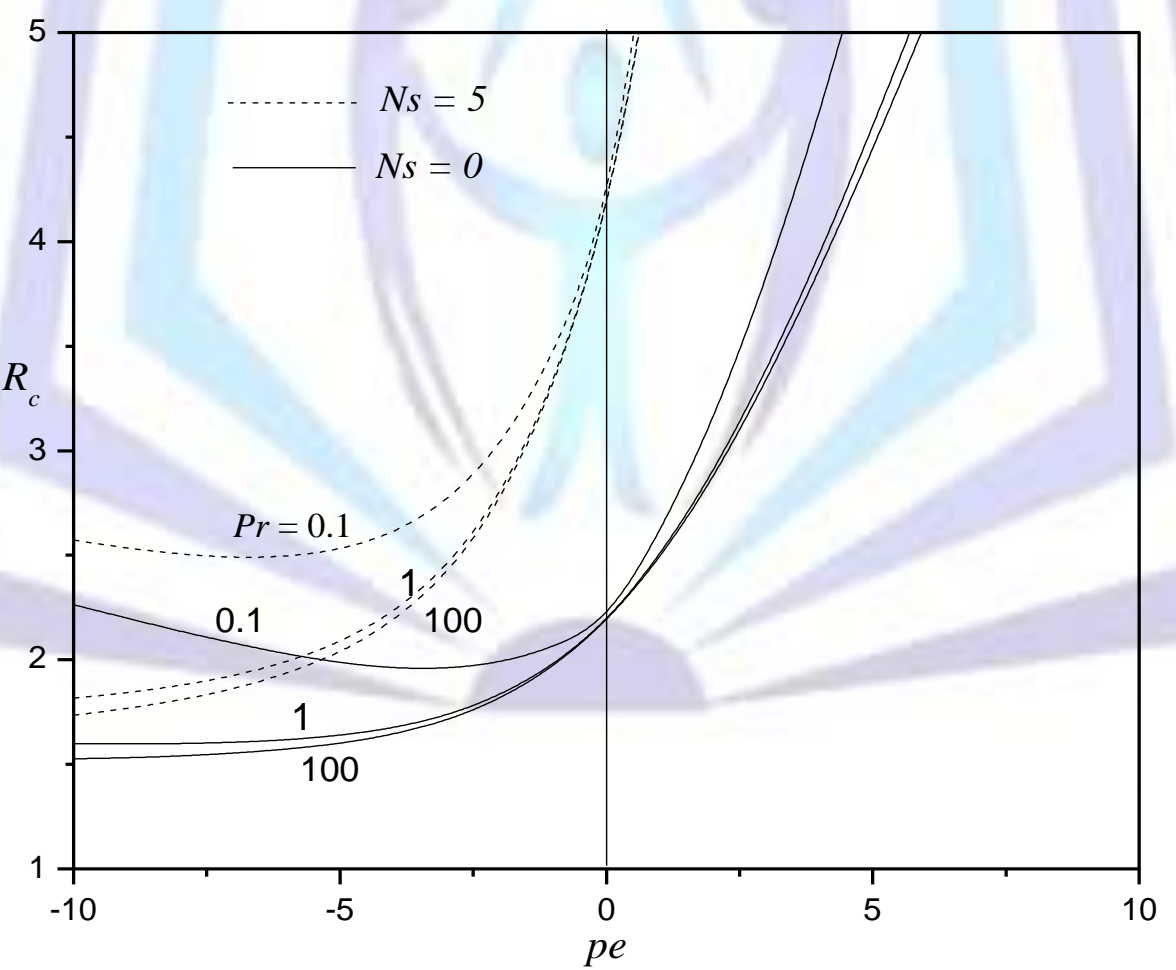

Fig.15 Critical Rayleigh number versus $P e$ for different values of $\operatorname{Pr}$ and $N s$ with $\lambda=1$ for lower boundary porous and insulating, upper boundary impermeable and insulating. 


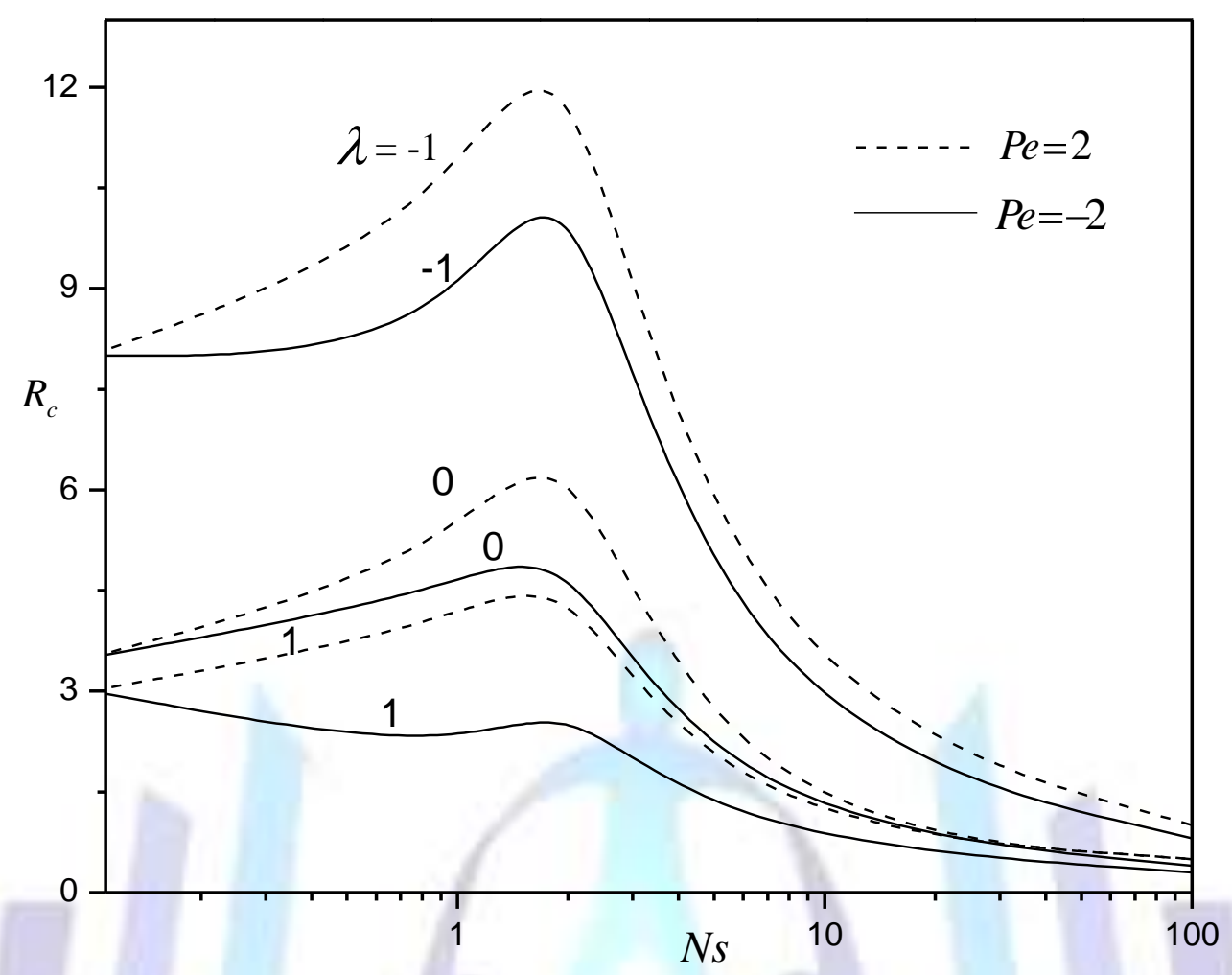

Fig. 16 Critical Rayleigh number versus $N s$ for different values of $\lambda$ and $P e$ with $\operatorname{Pr}=10$ for lower boundary porous and insulating, upper boundary impermeable and insulating.

\section{Case (2) Lower Boundary Impermeable and Insulating, Upper Boundary Porous and Insulating}

\section{(a)Absence of Variable Gravity $(\lambda=0)$}

The throughflow effects $P e$ and the dimensionless heat source strength $N s$, on the critical Rayleigh number $R_{c}$ are illustrated in Figs.7 and 8 respectively. From Figs.7 and 8 some unusual behaviours are observed namely, (i) upward throughflow shows destabilizing effect for $\operatorname{Pr}=1$ and 100 and the system initially shows some destabilizing effect for $P r=0.1$ when $N s=0$ (Fig. 7) (ii) both upward and downward throughflow shows stabilizing effect for all values of $P r$ when $N s=5$ (Fig. 7) and (iii) increasing internal heat source strength causes destabilizing effect for both upward and downward throughflow (Fig. 8).

\section{(b)Presence of Variable Gravity $(\lambda \neq 0)$}

Figures 9 and 10 exhibit plots of $R_{c}$ as a function of $P e$ for two values of variable gravity parameter $\lambda=-1,1$ respectively with three values of Prandtl number $\operatorname{Pr}=0.1,1$ and 100. From Figs. 9 and 10 it is observed that for all values of $\mathrm{Pr}$ the both upward and downward throughflow stabilizes the system in the presence of internal heating $(N s=5)$ for both values of $\lambda=-1$ and 1. In the absence of internal heating $(N s=0)$ the system stabilizes for downward throughflow for all values of $P r$ while, for upward throughflow the system is destabilizing when $0 \leq P e \leq 3.6,0 \leq P e \leq 5.6$ for $\operatorname{Pr}=0.1$ when $\lambda=-1,1$ respectively and for higher values of $P e$ the system is stabilizing and for $P r=1$ and 100 system is destabilizing. And also from Figs.9 and 10, it can be observed that, $R_{c}$ is maximum when the variable gravity parameter $\lambda$ takes the value -1 which is similar to case (1).

The effect of the variable gravity parameter $\lambda$ and the dimensionless heat source $N s$, on the critical Rayleigh number $R_{c}$ is illustrated in Fig.11for $P r=10$, and $P e=2,-2$. From Fig. 11 it is 
observed that increasing internal heat source strength causes destabilizing effect for both upward and downward throughflow for all three values of gravity parameter $\lambda$.

\section{Case (3) Lower Boundary Porous and Insulating, Upper Boundary Impermeable and Insulating}

\section{(a)Absence of Variable Gravity $(\lambda=0)$}

The throughflow effects $P e$ and the dimensionless heat source strength $N s$, on the critical Rayleigh number $R_{c}$ are illustrated in Figs.12 and 13 respectively. From Figs.12 and 13 some unusual behaviours are observed namely, (i) upward throughflow shows stabilizing effect for all values of $\operatorname{Pr}$ (Fig. 12) (ii) downward throughflow shows some destabilizing effect for $\operatorname{Pr}=0.1$ and system always destabilizes for $\operatorname{Pr}=1,100$ (Fig. 12) and (iii) increasing internal heat source strength causes stabilizing effect for both upward and downward throughflow initially (Fig. 13).

\section{(b)Presence of Variable Gravity $(\lambda \neq 0)$}

Figures 14 and 15 exhibit plots of $R_{c}$ as a function of $P e$ for two values of variable gravity parameter $\lambda=-1,1$ with three values of Prandtl number $\operatorname{Pr}=0.1,1$ and 100 .From Figs. 14 and 15 it is observed that for all values of $P r$ the both upward throughflow stabilizes in the absence $(N s=0)$ and presence $(N s=5)$ of internal heating for both values of $\lambda$ and downward throughflow destabilizes for $\operatorname{Pr}=1,100$. For $\operatorname{Pr}=0.1 \quad$ system destabilizing when $-5.8 \leq P e \leq 0,-6.2 \leq P e \leq 0$ for $N s=0,5$ respectively, when $\lambda=-1$ and $-3.6 \leq P e \leq 0,-8.4 \leq P e \leq 0$ for $N s=0,5$ respectively, when $\lambda=1$. And also from Figs.14 and 15, it can be observed that, $R_{c}$ is maximum when the variable gravity parameter $\lambda$ takes the value -1 which is also similar to above cases.

The effect of the variable gravity parameter $\lambda$ and the dimensionless heat source $N s$, on the critical Rayleigh number $R_{c}$ is illustrated in Fig. 16 for $\operatorname{Pr}=10$, and $\operatorname{Pe}=2,-2$. From Fig. 16 it is observed that increasing internal heat source strength causes stabilizing effect for both upward and downward throughflow initially for all three values of gravity parameter $\lambda$.

\section{Conclusions}

The onset of penetrative convection via internal heating in porous layer is studied in the presence of a vertical throughflow and variable gravity field. From the foregoing analysis, it is observed that the stability characteristics of the configuration depend crucially on (i) the presence of internal heating in the porous layer, (ii) variable gravity field, (iii) types of boundary conditions and (iv) throughflow direction. In absence of both gravity field and internal heating some usual behaviours are observed namely, (i) when the lower and upper boundaries are of same type, the critical Rayleigh number is an even function of $P e$ and system gets stabilized irrespective of direction of throughflow (ii) when the two boundaries are different types throughflow in one direction will be destabilize the system up to certain values of $P e$. However in the presence of gravity field and internal heating, throughflow in one particular direction destabilizes the system up to certain values of $P e$ for all the three types boundaries. The critical Rayleigh number is maximum when the variable gravity parameter $\lambda<0$ in all the three types boundaries. 
Table 1 Comparison of critical Rayleigh number with those of Nield [34] for case (1) and case (2) boundaries.

\begin{tabular}{|c|c|c|c|c|}
\hline \multirow{2}{*}{$P e$} & \multicolumn{2}{|c|}{ Nield [34] } & \multicolumn{2}{c|}{$\begin{array}{c}\text { Present study } \\
\lambda=0, N s=0 \text { and } G=0\end{array}$} \\
\cline { 2 - 5 } & Case1 & Case2 & Case1 & Case2 \\
\hline 0 & 12 & 3 & 12 & 3 \\
\hline 1.0 & 12.1986 & 2.6812 & 12.1986 & 2.6812 \\
\hline 2.0 & 12.7781 & 2.4599 & 12.7781 & 2.4599 \\
\hline 3.0 & 13.6947 & 2.3118 & 13.6947 & 2.3118 \\
\hline 4.0 & 14.8889 & 2.2148 & 14.8889 & 2.2148 \\
\hline 5.0 & 16.2981 & 2.1517 & 16.2981 & 2.1517 \\
\hline 10.0 & 24.9972 & 2.0407 & 24.9972 & 2.0407 \\
\hline
\end{tabular}

\section{References}

Alex, S. M., Patil, P. R. (1999). Convection in a Porous medium with inclined temperature gradient in a variable gravity field, J. Energy, Heat Mass Transfer, 21, 145-156.

Alex, S. M., Patil, P. R .(2002). Effect of a variable gravity field on thermal instability in a porous medium with inclined temperature gradient with inclined temperature gradient and vertical throughflow, J. Porous Media, 5 , 137-147.

Beck, J. L.(1972). Convection in a box of porous material saturated with fluid, Phys. Fluids. 15,1377- 1384.

Barletta, Eugenia Rossi di Schio, Leiv Storesletten.(2010). Convective roll instabilities of vertical throughflow with viscous dissipation in a horizontal porous layer, Transp. Porous Med. 461-477.

Carr, M.(2004). Penetrative convection in a superposed porous- medium-fluid layer via internal heating, J. Fluid Mech. 509, 305-329.

Chen, C. F., and Chen, F.(1992). Onset of salt finger convection in a gravity gradient, 4, 451-452.

Phys. Fluids A,

Clever, R., Schubert, G., and Busse, F. H.(1993). Two-Dimensional oscillatory convection in a gravitationally modulated fluid layer, J. Fluid Mech.253,663-680.

Carr, M., Putter, S.(2003). Penetrative convection in a horizontally isotropic porous layer, Continuum Mech. Thermodyn.1, 33-43. 
Govender, S.(2005). Linear stability and convection in a gravity modulated porous layer heated from below: transition from synchronous to subharmonic Solutions, Transport i Porous Media. 59, 227-238.

Hill, A. A.(2004). Penetrative convection induced by the absorption of radiation with nonlinear internal heat source, Dyn. Atmos. Oceans 38, 57-67, 2004.

Homsy, G. M., Sherwood, A. E.: Convective instability in porous with throughflow, AlChe J. 22,168-174.

Hill, A. A., Rionero, S., Straughan, B.(2007). Global stability for penetrative convection with throughflow in a porous material, IMA J. Appl. Math. 72, 635-643.

Hill, A. A.(2007). Unconditional nonlinear stability for convection in a porous medium with vertical throughflow, Acta Mech. 193,197-206.

Hill, A. A., Carr, M.(2010). Sharp global nonlinear stability for a fluid overlying a highly porous material, Proc. Roy. Soc. London A. 466, 127-140.

Khalili, A., Shivakumara, I. S.91998). on set of convection in porous layer with net throughflow and internal heat generation, Phys. Fluids, 10, 315-317.

Khalili, A., Shivakumara, I. S.(2003). Non-Darcian effects on the onset of convection in porous layer with throughflow, Transp. Porous Med. 53, 245-263.

Kaloni, P. N., Qiao, Z.(2001). Non-linear convection in a porous medium with inclined temperature and variable gravity effects, Int. J. Heat Mass Transf. 44, 1585-1591.

Nield, D. A.(1987). Convective instabilities in porous media with throughflow, AIChe J.33, 1222-1224.

Nield D. A., Kuznetsov, A. V.(2011). Onset of convection in a porous medium with strong vertical throughflow, Transp. Porous Med. doi:10.1007/s11242-011-9821, 2011.

Nield, D. A., Bejan, A.(2006). Convection in Porous Media, $3^{\text {rd }}$ Springer-Verlag. New York.

Nield, D. A., Joseph. D. D.(1985). Effect of quadratic drag on convection in a saturated porous medium, Phys. Fluids.28, 995-997.

Pradhan, G. K., and Samal, P. C.(1987). Thermal stability of a fluid layer under variable body forces, J. Math. Anal. Appl. 122, 487-495.

Prakash K. and Raj H.(1996). Rayleigh-Taylor instability of a partially ionized plasma in the presence of variable gravitational field in porous medium, J. Ind. Math. Soc. 62 163,800.

Rees, D. A. S., Pop, I. (2000) The effect of $g$-Jitter on vertical free convection boundary layer flow in porous media, Int. Commun. Heat Mass Transfer, 27, 415-424..

Rodot, H., Regel, L. L., and Turtchaninov, A. M.(1990). Crystal growth of IV-VI semiconductors in a centrifuge, J. Cryst. Growth, 104,280-284.

Rionero, S., Straughan, B.(1990). Convection in a porous medium with internal heat source and variable gravity effects, Int. J. Eng. Sci. 28, 497-503. 
Straughan, B.(1989). Convection in a variable gravity field, J. Math. Anal. Appl. 140,467-475.

Sutton, F. M.(1970). Onset of convection in a porous channel with net throughflow, Phys. Fluids 13, 19311934.

Shivakumara, I. S., Nanjundappa, C. E.(2006). Effects of quadratic drag and throughflow on double diffusive convection in a porous layer, Int. Comm. Heat Mass Transf. 33, 357-363.

Straughan, B.,(2008). Stability and wave motion in porous media, Appl. Math. Sci. Ser.vol. 165. Springer, New York.

Saunders, B. V., Murray, B. T., McFadden, G. B., Coriell, S. R., and Wheeler, A. A.(1992). The effect of gravity modulation on thermo solutal convection in an infinite layerof fluid, Phys. Fluids. 4, 1176-1189.

Straughan, B., Walker, D. W.(1996). Anisotropic porous penetrative convection, Proc. R.Soc. Lond. A 452, 97-115.

Tse, K. L., Chasnov, J. R.(1998). A Fourier Hermite pseudo spectral method for penetrative convection, J. Comput. Phys. 142, 489-505.

Zhang, K. K., Schubert, G.(2002). From penetrative convection to teleconvection, Astrophys. J. 572, 461476. 